\title{
ARTICLE OPEN Multiple unpinned Dirac points in group-Va single-layers with phosphorene structure
}

\author{
Yunhao Lu ${ }^{1,2}$, Di Zhou ${ }^{1}$, Guoqing Chang ${ }^{3,4}$, Shan Guan ${ }^{5}$, Weiguang Chen ${ }^{6}$, Yinzhu Jiang ${ }^{1,2}$, Jianzhong Jiang ${ }^{1,2}$, Xue-sen Wang $^{4}$, \\ Shengyuan A Yang ${ }^{5}$, Yuan Ping Feng ${ }^{4}$, Yoshiyuki Kawazoe ${ }^{7,8}$ and Hsin Lin $^{3,4}$
}

Emergent Dirac fermion states underlie many intriguing properties of graphene, and the search for them constitutes one strong motivation to explore two-dimensional (2D) allotropes of other elements. Phosphorene, the ultrathin layers of black phosphorous, has been a subject of intense investigations recently, and it was found that other group-Va elements could also form 2D layers with similar puckered lattice structure. Here, by a close examination of their electronic band structure evolution, we discover two types of Dirac fermion states emerging in the low-energy spectrum. One pair of (type-l) Dirac points is sitting on high-symmetry lines, while two pairs of (type-II) Dirac points are located at generic $k$-points, with different anisotropic dispersions determined by the reduced symmetries at their locations. Such fully-unpinned (type-II) 2D Dirac points are discovered for the first time. In the absence of spin-orbit coupling (SOC), we find that each Dirac node is protected by the sublattice symmetry from gap opening, which is in turn ensured by any one of three point group symmetries. The SOC generally gaps the Dirac nodes, and for the type-I case, this drives the system into a quantum spin Hall insulator phase. We suggest possible ways to realise the unpinned Dirac points in strained phosphorene.

npj Computational Materials (2016) 2, 16011; doi:10.1038/npjcompumats.2016.11; published online 10 June 2016

\section{INTRODUCTION}

Recent years have witnessed a surge of research interest in the study of Dirac fermions in condensed matter systems, ranging from graphene and topological insulator surfaces in two-dimensions (2D) to Dirac and Weyl semimetals in 3D, ${ }^{1-4}$ which possess many intriguing physical properties owing to their relativistic dispersion and chiral nature. Especially, 2D Dirac fermion states have been extensively discussed in honeycomb lattices, commonly shared by group-IVa elements with graphene as the most prominent example, ${ }^{5-9}$ for which Dirac points are pinned at the two inequivalent high-symmetry points $K$ and $K^{\prime}$ of the hexagonal Brillouin zone (BZ), around which the dispersion is linear and isotropic. Later on, 2D Dirac points on high-symmetry lines were also predicted in some nanostructured materials, ${ }^{10}$ including graphynes ${ }^{11}$ and rectangular carbon and boron allotropes. ${ }^{12,13}$ However, the possibility of 2D Dirac points at generic $k$-points has not been addressed, and such Dirac point has not been found so far.

Meanwhile, the exploration of 2D materials built of group-Va elements $(\mathrm{P}, \mathrm{As}, \mathrm{Sb}$ and $\mathrm{Bi})$ has just started. Single- and few-layer black phosphorous, known as phosphorene, have been successfully fabricated, and was shown to be semiconducting with a thickness-dependent bandgap and a good mobility up to $\sim 10^{3} \mathrm{~cm}^{2} / \mathrm{Vs}$, generating intense interest. ${ }^{14-21}$ While 2D allotropes with different lattice structures have been predicted and analysed for the other group-Va elements, ${ }^{22-25}$ we note that the puckered lattice structure similar to phosphorene has been demonstrated experimentally for Sb (refs 26-28; W. Xu et al., unpublished) and $\mathrm{Bi}^{29-32}$ (down to single-layer) grown on suitable substrates, and been predicted for As as well. ${ }^{22}$ Motivated by these previous experimental and theoretical works, and in view of the ubiquitous presence of the Dirac fermions and the associated interesting physics, one may wonder: Is it possible to have Dirac fermion states hosted in such 2D puckered lattices? A simple consideration shows that here any possible Dirac point cannot occur at high-symmetry points. The reason is that each Dirac point at $\boldsymbol{k}$ must have a time reversal (TR) partner at $-\boldsymbol{k}$ with opposite chirality, whereas the BZ of the puckered lattice has a rectangular shape, of which all the high-symmetry points are invariant under TR. Therefore, if Dirac states indeed exist in such systems, they must be of a type distinct from those in graphene.

In this work, we address the above question by investigating the electronic structures of group-Va 2D puckered lattices. We find that Dirac fermion states not only exist, but in fact occur with two different types: one type (referred to as type-l) of (two) Dirac points are located on high-symmetry lines; while the other type (referred to as type-II) of (four) Dirac points are located at generic $k$-points. Depending on their reduced symmetries, dispersions around these points exhibit different anisotropic behaviours. Points of each type can generate or annihilate in pairs of opposite chiralities, accompanying topological phase transitions from a band insulator to a 2D Dirac semimetal phase, and since they are not fixed at high-symmetry points, their locations can be moved around in the BZ. Particularly, to our best knowledge, the novel

\footnotetext{
${ }^{1}$ School of Materials Science and Engineering, Zhejiang University, Hangzhou, China; ${ }^{2}$ State Key Laboratory of Silicon Materials, Zhejiang University, Hangzhou, China; ${ }^{3}$ Centre for Advanced 2D Materials and Graphene Research Centre, National University of Singapore, Singapore, Singapore; ${ }^{4}$ Department of Physics, National University of Singapore,

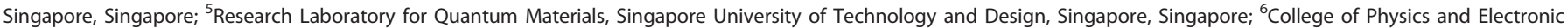

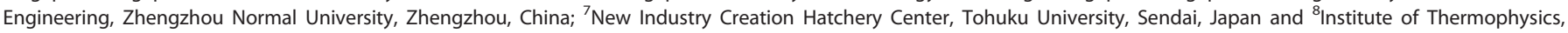
Siberian Branch of Russian Academy of Sciences, Novosibirsk, Russia.

Correspondence: Y Lu (luyh@zju.edu.cn) or SA Yang (shengyuan_yang@sutd.edu.sg) or H Lin (nilnish@gmail.com)

Received 2 December 2015; revised 20 April 2016; accepted 22 April 2016
} 
fully-unpinned (type-II) 2D Dirac points are discovered here for the first time. In the absence of spin-orbit coupling (SOC), each Dirac node is protected from gap opening by a sublattice (chiral) symmetry, which can in turn be ensured by any one of three point group symmetries. The inclusion of SOC could gap the Dirac nodes, and in the case of type-I nodes it transforms the system into a quantum spin Hall $(\mathrm{QSH})$ insulator phase. All these properties make the system distinct from graphene and other 2D materials. We further suggest that the novel unpinned Dirac points can be experimentally realized by the strain engineering of phosphorene. Our discovery therefore greatly advances our fundamental understanding of 2D Dirac points, and it also suggests a promising platform for exploring interesting effects with novel types of Dirac fermions.

\section{RESULTS}

A group-Va pnictogen atom typically forms three covalent bonds with its neighbours. As shown in Figure 1 for a single-layer phosphorene structure, the $\mathrm{P}$ atoms have strong $s p^{3}$-hybridisation character hence the three P-P bonds are more close to a tetrahedral configuration. This results in two atomic planes (marked with red and blue colours) having a vertical separation of $h$ comparable to the bond length. In each atomic plane, the bonding between atoms forms zig-zag chains along $y$-direction. The unit cell has a four-atom basis, which we label as $A_{U}, B_{U}, A_{\mathrm{L}}$ and $B_{\mathrm{L}}$ (see Figure $1 \mathrm{c}$ ), where $\mathrm{U}$ and $\mathrm{L}$ refer to the upper- and lower-plane, respectively. The structure has a non-symmorphic $D_{2 h}$ (7) space group which includes the following elements that will be important in our discussion: an inversion centre $i$, a vertical mirror plane $\sigma_{v}$ perpendicular to $\hat{y}$, and two twofold rotational axes $c_{2 y}$ and $c_{2 z}$. Note that due to the puckering of the layer, the mirror planes perpendicular to $\hat{x}$ and $\hat{z}$ are broken. With the same valence electron configuration, $\mathrm{As}, \mathrm{Sb}$ and $\mathrm{Bi}$ possess allotropes with similar puckered lattice structures.

To study the electronic properties, we performed first-principles calculations based on the density functional theory (DFT). The details are described in the materials and methods. The calculated geometric parameters of group-Va 2D puckered lattices with $D_{2 h}(7)$ symmetry are summarised in the Supplementary Information. The obtained structures agree with the experiments and other theoretical calculations (refs 17,22,32; W. Xu et al., unpublished). The lattice constants $a>b$, reflecting that the inter-chain coupling is weaker than the coupling along the zig-zag chains. The angle $\theta_{2}$ increases from $\sim 70^{\circ}$ for $P$ to $\sim 85^{\circ}$ for
$\mathrm{Bi}$, whereas $\theta_{1}$ remains $\sim 95^{\circ}$. The inter-plane separation $h$, as well as the bond lengths $R_{1}$ and $R_{3}$ increase by almost $1 \AA$; from $\mathrm{P}$ to $\mathrm{Bi}$, while $R_{2}$, the distance between sites of neighbouring zig-zag chains, increases only slightly, implying that the inter-chain coupling becomes relatively more important with increasing atomic number.

We first examine their corresponding band structures without SOC, whose effect will be discussed later. The results are shown in Figure 2. The puckered lattice of $P$ is a semiconductor with $a$ bandgap around $\Gamma$-point. From $\mathrm{P}$ to $\mathrm{Bi}$, the direct bandgap at $\Gamma$-point keeps decreasing, and a drastic change occurs from $\mathrm{Sb}$ to $\mathrm{Bi}$ where linear band crossings can be clearly spotted along the $\Gamma-X_{2}$ line. Examination of the band dispersion around the two points (labelled as $D$ and $D^{\prime}$ in Figure 1d) shows that they are indeed Dirac points (see Figure 3a). Furthermore, along $\Gamma-X_{1}$ line, there gradually appear two sharp local band extremum points for both conduction and valence bands, where the local gap decreases from $\mathrm{P}$ to $\mathrm{Sb}$ with the two bands almost touching for $\mathrm{Sb}$, yet the trend breaks for $\mathrm{Bi}$. Remarkably, close examination reveals that for $\mathrm{Sb}$ and $\mathrm{Bi}$, close to each extremum point there are actually two Dirac points on the two sides of the $\Gamma-X_{1}$ line (see Figures $1 \mathrm{~d}$ and $3 \mathrm{~b}$ ). The energy dispersions around these Dirac points are shown in Figures $1 \mathrm{e}$ and $\mathrm{f}$, clearly demonstrating the Dirac cone characters. Therefore, two types of Dirac points with distinct symmetry characters exist in this system: one pair of type-I Dirac points $\left(D\right.$ and $\left.D^{\prime}\right)$ sitting on high-symmetry lines and two pairs of type-II Dirac points (near $F$ and $F^{\prime}$ ) at generic $k$-points.

The band evolution around $\Gamma$-point from $\mathrm{Sb}$ to $\mathrm{Bi}$ and the appearance of type-I Dirac points in Figure 2 are reminiscent of a band-inversion process. Indeed, by checking the parity eigenvalues at $\Gamma$, one confirms that the band order is reversed for $\mathrm{Bi}$ around $\Gamma$-point (see Supplementary Information). For a better understanding, we construct a tight-binding model trying to capture the physics around $\Gamma$-point. Since the low-energy bands are dominated with $p_{z}$-orbital character (Figure 2), we take one orbital per site, and include couplings along $R_{1}$ and $R_{2}$ in the same atomic plane (with amplitudes $t_{1}$ and $t_{2}$, respectively), as well as nearest-neighbour inter-plane hopping along $R_{3}$ (with amplitude $t_{\perp}$ ) (see Supplementary Information). Written in the basis of $\left(A_{U}, A_{\mathrm{L}}, B_{\mathrm{U}}, B_{\mathrm{L}}\right)$, the Hamiltonian takes the form:

$$
\mathcal{H}(\boldsymbol{k})=\left[\begin{array}{cc}
0 & Q(\boldsymbol{k}) \\
Q^{\dagger}(\boldsymbol{k}) & 0
\end{array}\right]
$$

where $Q(\boldsymbol{k})$ is a $2 \times 2$ matrix of the Fourier transformed hopping terms (see Supplementary Information). The Hamiltonian (1) can a

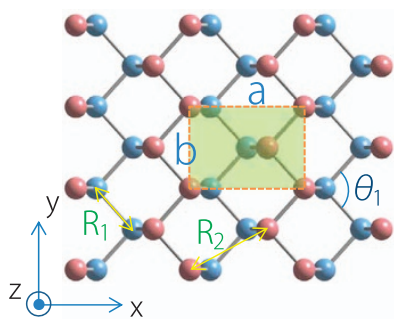

b

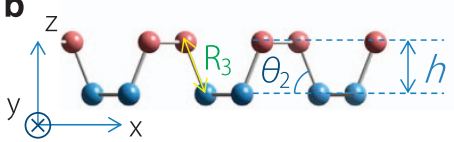

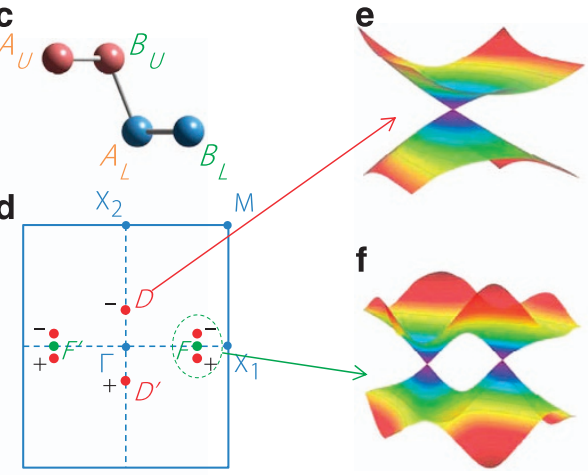

Figure 1. (a and b) Top- and side-view of the 2D puckered lattice structure. The green shaded region marks the unit cell. (c) Four-atom basis sites in a unit cell. (d) 2D Brillouin zone with high-symmetry points. The locations of Dirac points are schematically marked by the red dots: two type-I Dirac points at $D$ and $D^{\prime}$ on $\Gamma-X_{2}$; and four type-II Dirac points around $\Gamma-X_{1}$ forming two mirror image pairs. $F\left(F^{\prime}\right)$ on $\Gamma-X_{1}$ labels the mid-point of each pair. $+(-)$ indicates the chirality of each point. e and $\mathbf{f}$ show the schematic energy dispersions around point $D$ and point $F$, respectively, corresponding to the result in Figure 3. 


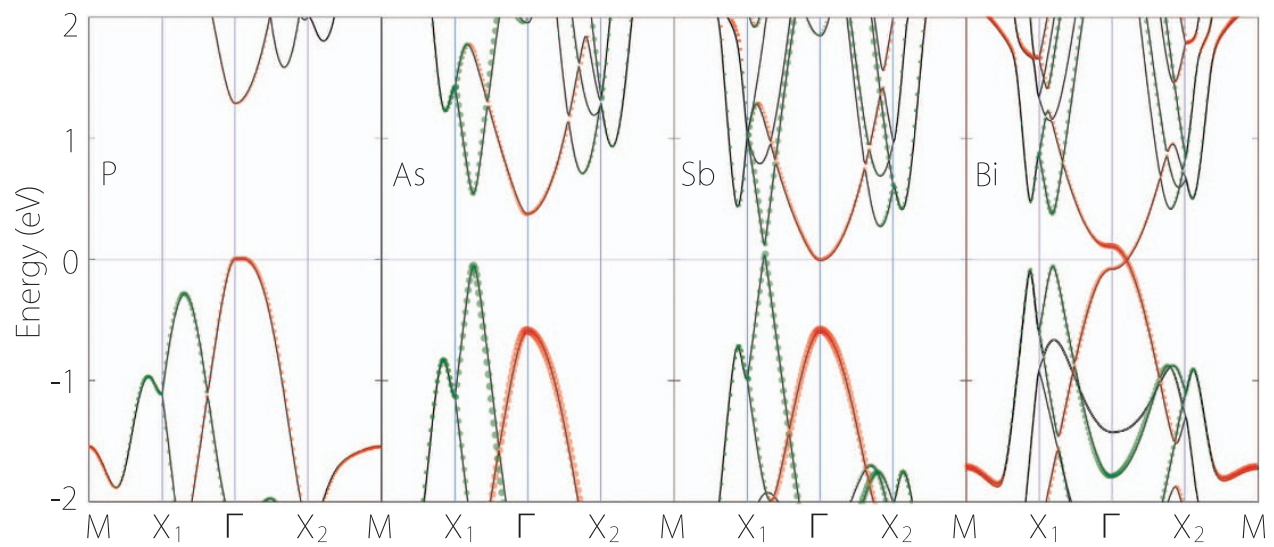

Figure 2. Band structures of group-Va elements with 2D phosphorene lattice structures in the absence of SOC. The size of red (green) dots denotes the weight of projection onto $p_{z}\left(p_{x}\right)$ atomic orbitals.
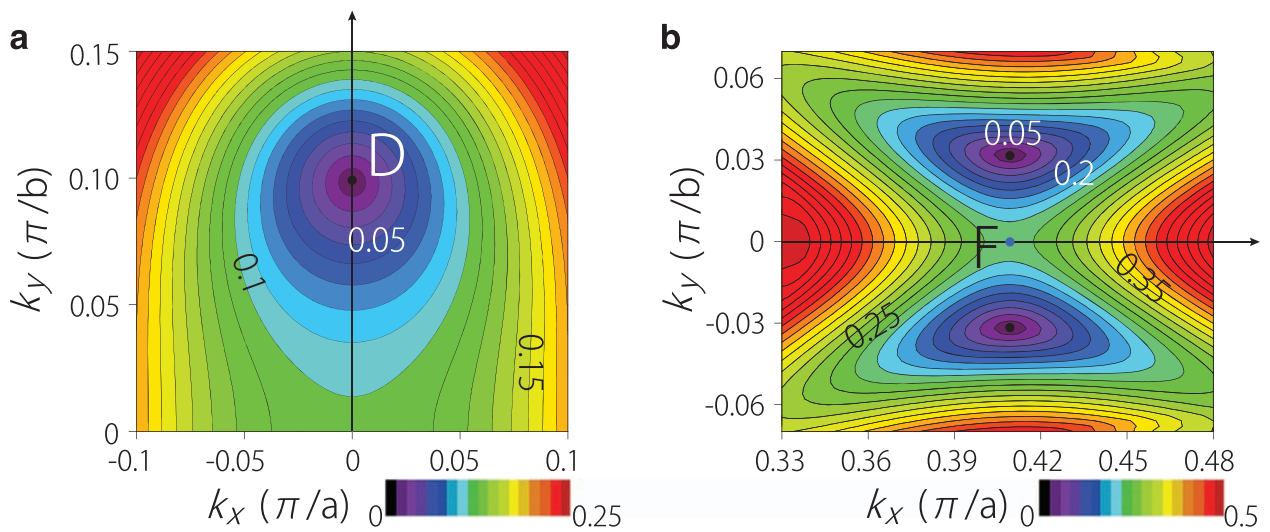

Figure 3. Dispersion around (a) type-I and (b) type-II Dirac points for 2D puckered Bi. Black dots mark the Dirac point locations. Energy is in unit of eV.

be diagonalized and possible band crossings can be probed by searching for the zero-energy modes, which exist when the condition $\lambda \equiv t_{\perp} /\left[2\left(t_{1}+t_{2}\right)\right]<1$ is satisfied, with two band touching points at $\left(0, \pm k_{\mathrm{D}}\right)$ where $k_{\mathrm{D}}=(2 / b) \arccos (\lambda)$. The direct gap at $\Gamma$ can be obtained as $\Delta=2\left[t_{\perp}-2\left(t_{1}+t_{2}\right)\right]$. Hence this simple model indeed captures the emergence of two Dirac points $D$ and $D^{\prime}$, along with a transition as parameter $\lambda$ varies: when $\lambda>1$, the system is a band insulator; when $\lambda<1$, it is a 2D Dirac semimetal. The transition occurs at the critical value $\lambda_{c}=1$ when the conduction and valence bands touch at $\Gamma$-point and the band order starts to be inverted. This corresponds to a quantum (and topological) phase transition, ${ }^{33}$ during which there is no symmetry change of the system.

Equation (1) captures the trend observed in DFT results. The overlap between- $p_{z}$ orbitals is larger along the $R_{3}$ bond, hence one expects that $t_{\perp}>t_{1}>t_{2}$. By fitting the DFT bands around $\Gamma$-point, one finds that from $\mathrm{P}$ to $\mathrm{Bi}, t_{\perp}$ decreases a lot, while $t_{2}$ increases and becomes relatively more important (Supplementary Information). The result shows that $\left(t_{\perp}, t_{1}, t_{2}\right)$ changes from $(2.50,0.77,0.33)$ for $\mathrm{Sb}$ to $(1.86,0.63,0.35)$ for Bi (units in eV). Hence $\lambda$ crosses the critical value from $\mathrm{Sb}$ to $\mathrm{Bi}$, indicating the band inversion at $\Gamma$ and the appearance of two Dirac points.

The emergence of low-energy relativistic chiral modes is the most remarkable property of Dirac points. ${ }^{33}$ To explicitly demonstrate this, we expand Hamiltonian (1) around each Dirac point, which leads to the low-energy Hamiltonian

$$
H_{\tau}(\boldsymbol{q})=v_{x} q_{x} \sigma_{y}+\tau v_{y} q_{y} \sigma_{x},
$$

where $\boldsymbol{q}$ is the wave-vector measured from each Dirac point, $\tau=$ \pm 1 for $D$ and $D^{\prime}, \sigma_{i}$ s are Pauli matrices for the sub-space spanned by the two eigenstates at the Dirac point (apart from the Bloch phase factor): $\left|u_{1}\right\rangle=(0,0,1,-1) / \sqrt{2}$ and $\left|u_{2}\right\rangle=(1,-1,0,0) / \sqrt{2}$ and $v_{x}=a t_{\perp}\left(t_{1}-t_{2}\right) /\left(t_{1}+t_{2}\right)$ and $v_{y}=b \sqrt{4\left(t_{1}+t_{2}\right)^{2}-t_{\perp}^{2}}$ are the two Fermi velocities. The form of equation (2) may also be argued solely from symmetry. Compared with graphene, these type-I points are unpinned from the high-symmetry points. They can be shifted along $\Gamma-X_{2}$ (and even pair-annihilated) by varying system parameters such as $\lambda$, although they cannot go off the line as constrained by the symmetries. In addition, different from graphene, ${ }^{6}$ the dispersion here is anisotropic, characterised by two different Fermi velocities.

Next, we turn to the fully-unpinned type-II Dirac points. The four type-II Dirac points start to appear for Sb in our DFT result, located close to the $\Gamma-X_{1}$ line. They can be more clearly seen for $\mathrm{Bi}$ (see Figure $3 b$ ). Again the band evolution implies a local band inversion near $F$ and $F^{\prime}$. Here $F$ and $F^{\prime}$ (on $\Gamma-X_{1}$ ) are the mid-points of the lines connecting each pair of the type-II points. The low-energy bands are mainly of $p_{x}$-orbital character. To reproduce the fine features using a tight-binding model would require more hopping terms. Instead, we construct a low-energy effective Hamiltonian around point $F\left(F^{\prime}\right)$ based on symmetry analysis. There the Hamiltonian is constrained by $\sigma_{v}$, which maps inside each pair (labelled by $\mu= \pm 1$ for $F$ and $F^{\prime}$ ), and by $i, c_{2 y}, c_{2 z}$, and TR that map between the two pairs. Expansion to leading order in each wave-vector component $q_{i}$ gives (see Supplementary 
4

Information)

$$
H_{\mu}(\boldsymbol{q})=w q_{x} \sigma_{y}+\left(-m_{0}+\mu w^{\prime} q_{x}+m_{1} q_{y}^{2}\right) \sigma_{x},
$$

where $\boldsymbol{q}$ is measured from $F$ (or $F^{\prime}$ ), $w, w^{\prime}, m_{0}$ and $m_{1}$ are expansion coefficients. Two Dirac points appear at $\left(0, \pm q_{0}\right)$ with $q_{0}=\sqrt{m_{0} / m_{1}}$ when $\operatorname{sgn}\left(m_{0} / m_{1}\right)=1$, corresponding to a local band inversion around $\boldsymbol{q}=0$. Further expansion of the Hamiltonian around the Dirac point $\left(0, v q_{0}\right)(v= \pm 1)$ leads to

$$
\tilde{H}_{\mu}^{\nu}=w q_{x} \sigma_{y}+\left[2 \nu q_{0}\left(q_{y}-\nu q_{0}\right)+\mu w^{\prime} q_{x}\right] \sigma_{x} \text {. }
$$

This demonstrates that the two points at $v= \pm 1$ are of opposite chirality, as required by $\sigma_{v}$. The dispersion is highly anisotropic (at leading order, characterised by three parameters: $w, q_{0}$ and $w^{\prime}$ ) because the Dirac point is at a generic $k$-point with less symmetry constraint, as compared with type-I Dirac points.

Unlike in 3D systems, Dirac nodes in 2D have a co-dimension of 2 hence are generally not protected from gap opening. ${ }^{33}$ In the absence of SOC, however, the Dirac nodes here are stable due to the protection by sublattice (chiral) symmetry between $\left\{A_{i}\right\}$ and $\left\{B_{i}\right\}$ $(i=U, L)$ sites, which allows the definition of a winding number ${ }^{34,35}$ (that is, quantised Berry phase in units of $\pi$ ) along a closed loop $\ell$ encircling each Dirac point: $N_{\ell}=\oint_{\ell} \mathcal{A}_{\boldsymbol{k}} \cdot \mathrm{d} \boldsymbol{k} / \pi= \pm 1$, where $\mathcal{A}_{\boldsymbol{k}}$ is the Berry connection of the occupied valence bands. And for a 2D Dirac point, the sign of $N_{\ell}$ (or the $\pm \pi$ Berry phase) is also referred to as the chirality. ${ }^{6}$ Using DFT results, we numerically calculate the Berry phase for each Dirac point and indeed confirm that they are quantised as $\pm \pi$. The signs are indicated in Figure $1 \mathrm{~d}$.

More interestingly, in the puckered lattice with a four-atom basis in a non-coplanar geometry, the sublattice symmetry can be ensured by any one of three independent point group symmetries: $i, c_{2 y}$ and $c_{2 z}$. The resulting protection of Dirac nodes can be explicitly demonstrated in low-energy models. For example, consider the type-I points described by equation (2). There the representations of $i, c_{2 y}$ and $c_{2 z}$ (denoted by $\mathcal{P}, \mathcal{R}_{y}$ and $\mathcal{R}_{z}$, respectively) are the same, which is, $\sigma_{x}$. Then the symmetry requirement $\mathcal{R}_{y} H_{\tau}\left(q_{x}, q_{y}\right) \mathcal{R}_{y}^{-1}=H_{\tau}\left(-q_{x}, q_{y}\right)$ by $c_{2 y}$ directly forbids the presence of a mass term $m \sigma_{z}$. Meanwhile, since $i$ and $c_{2 z}$ map one valley to the other, they protect the Dirac nodes when combined with TR (or $\sigma_{v}$ if it is unbroken), e.g., considering the combined symmetry of $c_{2 z}$ and TR (with representation $\mathcal{T}=K$ the complex conjugation operator): $\left(\mathcal{R}_{Z} \mathcal{T}\right) H_{\tau}(\boldsymbol{q})\left(\mathcal{R}_{Z} \mathcal{T}\right)^{-1}=H_{\tau}(\boldsymbol{q})$, which again forbids a mass generation. The underlying reason $i, c_{2 y}$ and $c_{2 z}$ each protects the Dirac node is that they each map between the two sublattices hence ensures the sublattice (chiral) symmetry. In comparison, the mirror plane $\sigma_{v}$ maps inside each sublattice, hence it alone cannot provide such protection. This reasoning is general and applies to type-ll points as well. (In equation (3), i, $c_{2 y}$ and $c_{2 z}$ have representations as $\sigma_{x}$ by construction, and when combined with $\mathrm{TR}$, again each forbids the generation of a mass term $\sim m \sigma_{z}$. See Supplementary Information.) We stress that the three symmetries $i, c_{2 y}$ and $c_{2 z}$ each protects the Dirac points independent of the other two. For example, we could disturb the system as in Figure 4 such that only one of the three symmetries survives. The corresponding DFT results confirm that the Dirac nodes still exist. Thus the crystalline symmetries actually offer multiple protections for the Dirac nodes in the current system.

SOC could break the sublattice symmetry. Hence when SOC is included, the Dirac nodes would generally be gapped. ${ }^{36}$ For type-I points, treating SOC as a perturbation, its leading-order symmetry-allowed form is $H_{\mathrm{SOC}}=\tau \Delta \sigma_{z} s_{z}$, where $s_{z}$ is Pauli matrix for real spin. This is similar to the intrinsic SOC term in graphene, ${ }^{37}$ which opens a gap of $2|\Delta|$ at the Dirac points. For the type-II points, we obtain $H_{\mathrm{SOC}}=\eta q_{y} \sigma_{z} s_{z}$ in equation (3) hence a gap of $2 q_{0}|\eta|$ is also opened at these Dirac points. Gap opening by SOC is closely related to the QSH insulator phase. ${ }^{1,2,37}$ Here the band topology can be directly deduced from the parity analysis at the four TR invariant momenta. ${ }^{38}$ This means that only the band inversion at $\Gamma$ between the two type-I points contributes to a non-trivial $\mathbb{Z}_{2}$ invariant; whereas that associated with type-II points does not. It follows that $\mathrm{Sb}$ is topologically trivial since it has only type-II Dirac points, while Bi is non-trivial since it has additional type-I points. These results are in agreement with previous studies. $^{32}$

Breaking all three symmetries $i, c_{2 y}$ and $c_{2 z}$ can also generate a trivial gap term $m \sigma_{z}$ at the Dirac points, which competes with the SOC gap. For example, this happens when each atomic plane forms additional buckling structure. ${ }^{32}$ Nevertheless, as long as the a

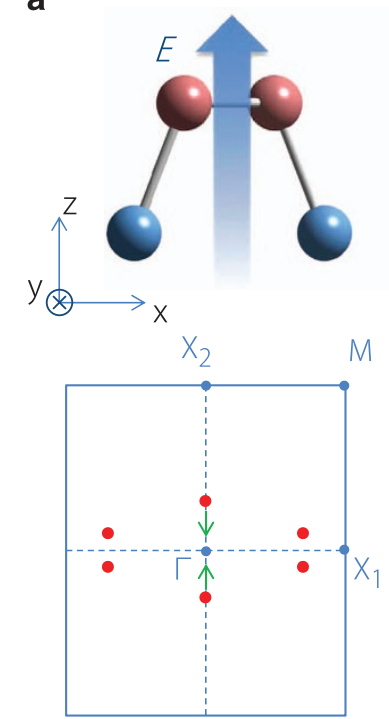

b

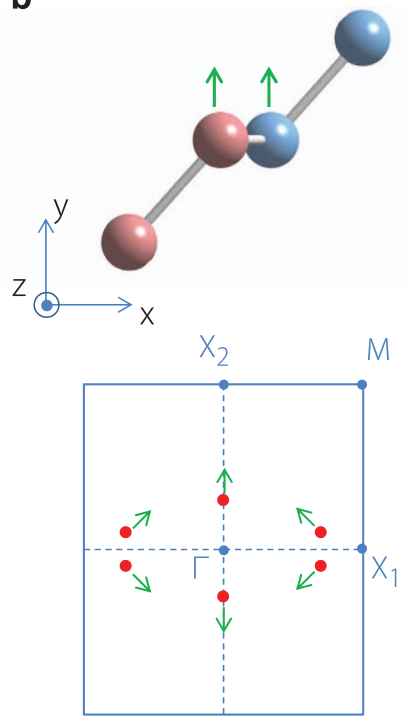

C

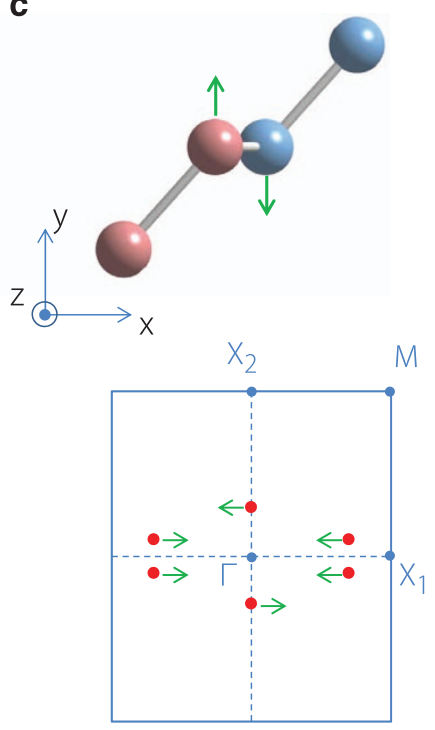

Figure 4. Perturbations which leave only one of the three symmetries preserved: (a) $c_{2 z}$ survives when applying $E$ field in $z$-direction; (b) $c_{2 y}$ survives when shifting the two basis sites along $\hat{y}$ in the same direction; (c) $i$ survives when shifting the two sites along $\hat{y}$ but in opposite directions. For each case, the Dirac nodes are still protected in the absence of SOC. The lower panel in each sub-figure schematically indicates the movement of the Dirac points under each perturbation. For the case in a, the type-ll points do not show appreciable change in their locations under a weak electric field, while the two type-I points annihilate with each other when they meet at r-point. 
trivial mass term does not close the SOC-induced gap, by adiabatic continuation the band topology will not change.

\section{DISCUSSION}

Due to their different locations and the associated symmetries, the two types of Dirac points here exhibit properties distinct from that of graphene. With preserved sublattice symmetry and in the absence of SOC, the Dirac nodes are topologically stable-they can only disappear by pair-annihilation between opposite chiralities. This is unlikely for graphene since the Dirac points are pinned at the high-symmetry points. In contrast, the two types of Dirac points here are less constrained. Pair-annihilation (pair-generation) indeed occurs during the quantum phase transition as observed from the band evolution.

It is noted that similar type-I points were also predicted in a few nanostructured materials. ${ }^{11-13}$ Meanwhile the type-II points discovered here are completely new. They are fully-unpinned and have highly anisotropic dispersions. With this discovery, now we can have an almost complete picture: 2D Dirac points can occur at high-symmetry points, along high-symmetry lines and also at generic $k$-points.

It is possible to have Dirac points, originally sitting at high-symmetry points, to become unpinned when crystalline symmetry is reduced due to structural distortions. However, we stress that the type-Il points here are distinct in that they are realized in a native crystalline structure with relatively high symmetry. Only in such a case, we can have a sharp contrast between generic $k$-points where the group of wave vectors is trivial and the high-symmetry $k$-points where the group is non-trivial, and accordingly the type-Il point can move around (hence fully-unpinned) without any symmetry-breaking. More importantly, it is just because that type-II points occur in a state with high symmetry that the Dirac nodes can be protected (in the absence of SOC): as we discussed, the various crystalline symmetries ensure the protection of the Dirac nodes from gap opening.

It is remarkable that the two different types of Dirac points can coexist in the same 2D material. We emphasise that it is a result of the lattice structure and the valence character of the pnictogen elements. Our DFT result indeed shows that even starting from the P lattice, the two types of Dirac points can be separately tuned to appear or disappear by lattice deformations. For example, we find that the type-II Dirac points can be generated in phosphorene by applying uniaxial tensile strains along the $y$-direction. The DFT result in Figures 5 and 6 indeed shows the band inversion on $\Gamma-X_{1}$ and the formation of four type-II Dirac points around a strain of $16 \%$. Since phosphorene has excellent mechanical properties and

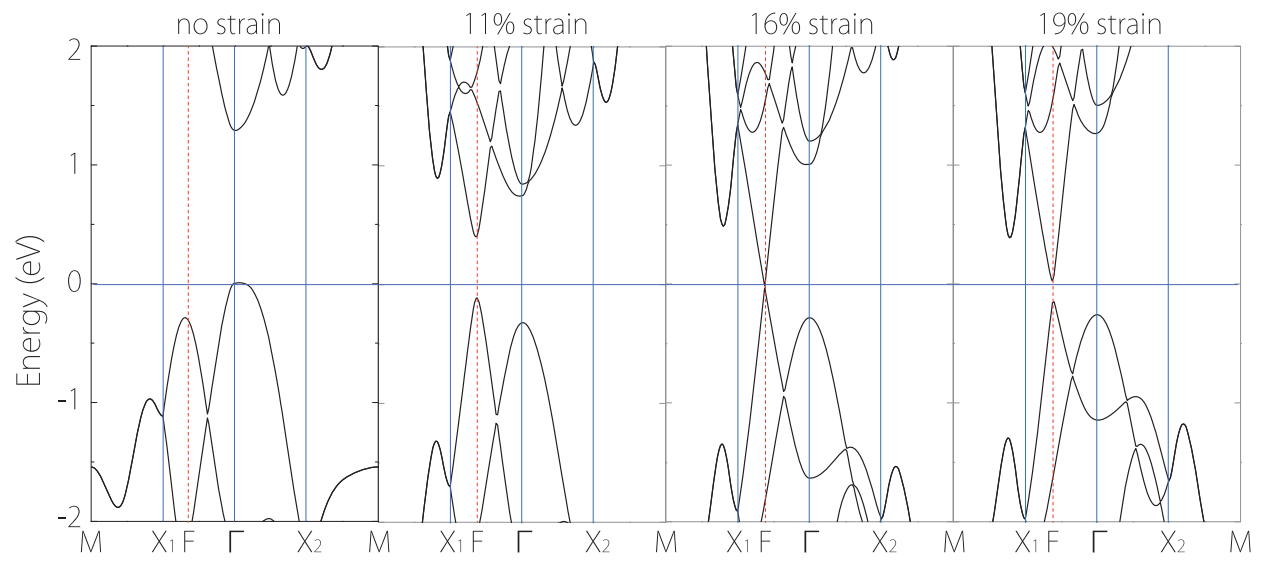

Figure 5. Band structure of phosphorene with uniaxial tensile strain applied along $y$-direction. Band inversion along $\Gamma-X_{1}$ line occurs around a value of $16 \%$.

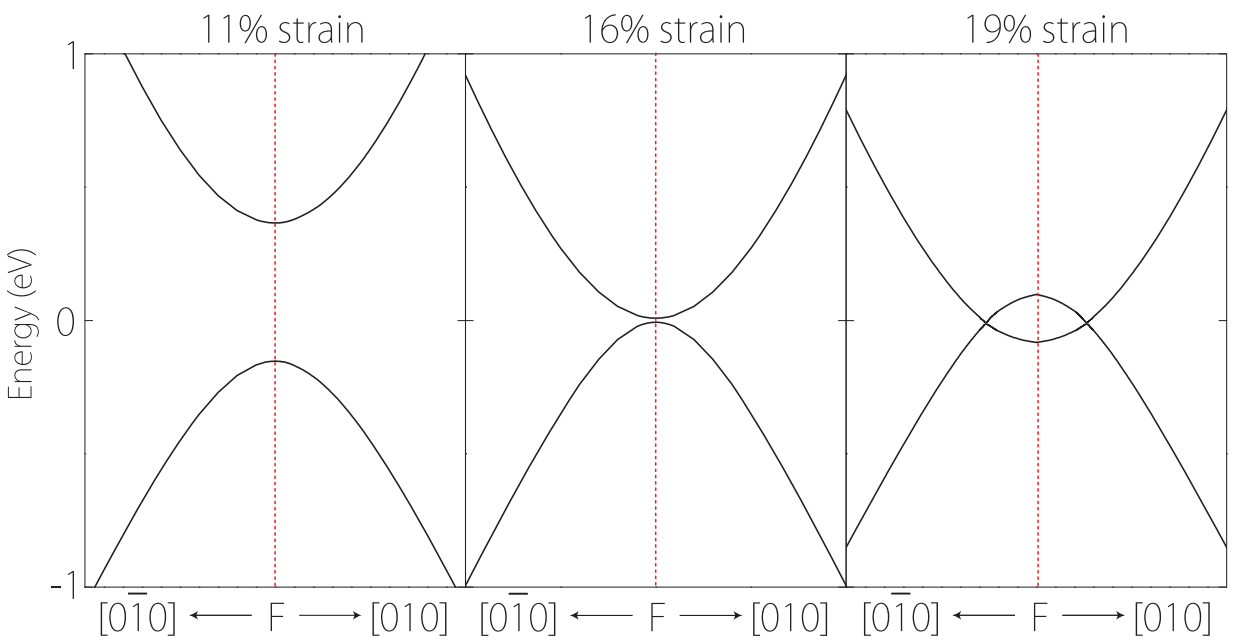

Figure 6. Band structure of phosphorene with uniaxial tensile strain applied along $y$-direction. Here the dispersion is plotted along a path crossing point $F$ (as indicated in Figure 5 ) and perpendicular to the $\Gamma-X_{1}$ direction. Two type-ll Dirac points near $F$ can be clearly observed at strain value $>16 \%$. 
a critical strains $>25 \%$ has been predicted, ${ }^{39}$ it is promising that the novel strain-induced topological phase transitions and the appearance of type-II Dirac points can be directly observed in strained phosphorene. The lattice deformation that produces type-I points is discussed in the Supplementary Information. Similar scenarios occur for other group-Va elements as well.

So far, single-layer $\mathrm{As}, \mathrm{Sb}$ and $\mathrm{Bi}$ in their free-standing form have not been realized yet. Nevertheless, in view of the rapid progress in experimental techniques, we expect that these materials could be fabricated in the near future. Especially, for $\mathrm{Sb}$ and $\mathrm{Bi}$, the puckered structures have been demonstrated by PVD growth down to single-layer thickness on suitable substrates (refs 26-32; W. Xu et al., unpublished). Besides the topological properties, the presence of Dirac states is expected to endow these 2D materials with many intriguing properties for applications, such as the very high mobility, the half-quantised quantum Hall effect, ${ }^{40}$ the universal optical absorption ${ }^{41}$ and etc. Due to the highly anisotropic dispersions of these new Dirac points, the electronic transport properties such as the conductivities would show strong direction dependence. In addition, since there is no symmetry connection between the two types of Dirac points, when they are both present, it is possible to independently shift each type of points relative to the Fermi level, e.g., by strain engineering, leading to self-doping and even the interesting scenario with both electron-like and hole-like Dirac fermions in the same system. With the multiple Dirac points with different chiralities, it is possible to further control the carriers near different Dirac points for valleytronic applications.

In conclusion, based on first-principles calculations of 2D allotropes of group-Va elements with puckered lattice structure, we predict the coexistence of two different types of Dirac points: Dirac points on high-symmetry lines and at generic $k$-points. In particular, the 2D Dirac points at generic $k$-points are fully-unpinned, have highly anisotropic dispersions and are discovered here for the first time. Combined with low-energy effective modelling, we unveil the low-energy properties of these Dirac points. We show that their appearance is associated with the band-inversion process corresponding to a topological phase transition. The topology/symmetry protection of the Dirac nodes is analysed in detail. Interestingly, because of the unique lattice structure, there is a triple-protection of the nodes by three independent point group symmetries. This also implies versatile methods to control the locations, as well as the dispersions around the Dirac points. When SOC is strong, the Dirac nodes are gapped, and in the case of type-I points (such as for $\mathrm{Bi}$ ) this drives the system into a QSH insulator phase. We further show that the topological phase transition and the novel unpinned Dirac points can be realized in strained phosphorene. Our work represents a significant conceptual advance in our fundamental understanding of 2D Dirac points. The result also suggests a new platform to explore novel types of 2D Dirac fermions both for their fascinating fundamental properties and for their promising electronic and valleytronic applications.

\section{MATERIALS AND METHODS}

\section{First-principles calculations}

Our first-principles calculations are based on the DFT implemented in the Vienna $a b$ initio simulation package. ${ }^{42}$ The projector augmented wave pseudopotential method is employed to model ionic potentials. ${ }^{43}$ Kinetic energy cutoff is set to $400 \mathrm{eV}$ and $k$-point sampling on the rectangular BZ is with a mesh size $20 \times 20$. The minimum vacuum layer thickness is $>20 \AA$; which is large enough to avoid artificial interactions with system images. The structure optimisation process is performed including SOC with the local density approximation for the exchange-correlation energy ${ }^{44}$ and with van der Waals corrections in the Grimme implementation. ${ }^{45}$ The force convergence criteria is set to be $0.01 \mathrm{eV} / \mathrm{A}$. Hybrid functional $(\text { HSE06) })^{46}$ is used for the band structure calculations.

\section{ACKNOWLEDGEMENTS}

The authors thank D.L. Deng and Shengli Zhang for helpful discussions. This work was supported by NSFC (Grant No. 11374009, 61574123 and 21373184), the National Key Basic Research Program of China (2012CB825700), SUTD-SRG-EPD2013062, Singapore MOE Academic Research Fund Tier 1 (SUTD-T1-2015004), A*STAR SERC 122-PSF-0017 and AcRF R-144-000-310-112. H.L. acknowledges support by Singapore National Research Foundation under NRF Award No. NRF-NRFF2013-03. Y.L. acknowledges Special Program for Applied Research on Super Computation of the NSFC-Guangdong Joint Fund (the second phase). The authors gratefully acknowledge support from SR16000 supercomputing resources of the Center for Computational Materials Science, Tohoku University.

\section{CONTRIBUTIONS}

Y.L. and D.Z. performed the first-principles calculations. G.C. and S.G. helped with the data analysis and model fitting. H.L. and S.A.Y. did the analytical modelling and symmetry/topology analysis. W.C., Y.J., J.J. and X.-s.W. participated in the discussion and analysis. X.-s.W., Y.P.F., Y.K., Y.L., S.A.Y. and H.L. supervised the work. Y.L., S.G. and S.A.Y. prepared the manuscript. All authors reviewed the manuscript.

\section{COMPETING INTERESTS}

The authors declare no conflict of interest.

\section{REFERENCES}

1. Hasan, M. Z. \& Kane, C. L. Colloquium: topological insulators. Rev. Mod. Phys. 82, 3045 (2010).

2. Qi, X.-L. \& Zhang, S.-C. Topological insulators and superconductors. Rev. Mod. Phys. 83, 1057 (2011).

3. Wan, X., Turner, A. M., Vishwanath, A. \& Savrasov, S. Y. Topological semimetal and fermi-arc surface states in the electronic structure of pyrochlore iridates. Phys. Rev. B 83, 205101 (2011).

4. Young, S.-M. et al. Dirac semimetal in three dimensions. Phys. Rev. Lett. 108, 140405 (2012).

5. Novoselov, K. S. et al. Electric field effect in atomically thin carbon films. Science 306, 666-669 (2004).

6. Castro Neto, A. H., Guinea, F., Peres, N. M. R., Novoselov, K. S. \& Geim, A. K. The electronic properties of graphene. Rev. Mod. Phys. 81, 1095 (2009).

7. Cahangirov, S., Topsakal, M., Akturk, E., Sahin, H. \& Ciraci, S. Two- and one-dimensional honeycomb structures of silicon and germanium. Phys. Rev. Lett. 102, 236804 (2009).

8. Liu, C.-C., Feng, W. \& Yao, Y. Quantum spin Hall effect in silicene and two-dimensional germanium. Phys. Rev. Lett. 107, 076802 (2011).

9. Xu, Y. et al. Large-gap quantum spin Hall insulators in tin films. Phys. Rev. Lett. 111, 136804 (2013).

10. See Wang J., Deng S., Liu Z. \& Liu Z. The rare two-dimensional materials with Dirac cones. Nat. Sci. Rev 2015; 2: 22.

11. Malko, D., Neiss, C., Vines, F. \& Görling, A. Competition for graphene: graphynes with direction-dependent dirac cones. Phys. Rev. Lett. 108, 086804 (2012).

12. Xu, L.-C. et al. Two dimensional Dirac carbon allotropes from graphene. Nanoscale 6, 1113 (2014).

13. Zhou, X.-F. et al. Semimetallic two-dimensional boron allotrope with massless Dirac fermions. Phys. Rev. Lett. 112, 085502 (2014).

14. Li, L. et al. Black phosphorus field-effect transistors. Nat. Nanotechnol. 9, 372 (2014).

15. Xia, F., Wang, H. \& Jia, Y. Rediscovering black phosphorus as an anisotropic layered material for optoelectronics and electronics. Nat. Commun. 5, 4458 (2014).

16. Liu, H. et al. Phosphorene: An unexplored 2D semiconductor with a high hole mobility. ACS Nano 8, 4033 (2014).

17. Qiao, J., Kong, X., Hu, Z.-X., Yang, F. \& Ji, W. High-mobility transport anisotropy and linear dichroism in few-layer black phosphorus. Nat. Commun. 5, 4475 (2014).

18. Rudenko, A. N. \& Katsnelson, M. I. Quasiparticle band structure and tight-binding model for single-and bilayer black phosphorus. Phys. Rev. B 89, 201408 (2014).

19. Liu, Q., Zhang, X., Abdalla, L. B., Fazzio, A. \& Zunger, A. Switching a normal insulator into a topological insulator via electric field with application to phosphorene. Nano Lett. 15, 1222 (2015).

20. Kim, J. et al. Observation of tunable band gap and anisotropic Dirac semimetal state in black phosphorus. Science 349, 723 (2015).

21. Woomer, A. H. et al. Phosphorene: Synthesis, scale-up, and quantitative optical spectroscopy. ACS Nano 9, 8869 (2015).

22. Kamal, C. \& Ezawa, M. Arsenene: Two-dimensional buckled and puckered honeycomb arsenic systems. Phys. Rev. B 91, 085423 (2015). 
23. Zhu, Z. \& Tomanek, D. Semiconducting layered blue phosphorus: a computational study. Phys. Rev. Lett. 112, 176802 (2014).

24. Zhang, S., Yan, Z., Li, Y., Chen, Z. \& Zeng, H. Atomically thin arsenene and antimonene: semimetal-semiconductor and indirect-direct band-gap transitions. Angew. Chem. Int. Ed. 54, 3112 (2015).

25. Wang, G., Pandey, R. \& Karna, S. P. Atomically thin group V elemental films: theoretical investigations of antimonene allotropes. ACS Appl. Mater. Interfaces 7, 11490 (2015)

26. Wang, X.-S., Kushvaha, S. S., Yan, Z. \& Xiao, W. Self-assembly of antimony nanowires on graphite. Appl. Phys. Lett. 88, 233105 (2006).

27. Bianchi, M. et al. Surface states on a topologically nontrivial semimetal: the case of Sb (110). Phys. Rev. B 85, 155431 (2012).

28. Strozecka, A. et al. Unconventional spin texture of a topologically nontrivial semimetal Sb (110). N. J. Phys. 14, 103026 (2012).

29. Nagao, T. et al. Nanofilm allotrope and phase transformation of ultrathin Bi film on Si(111)-77. Phys. Rev. Lett. 93, 105501 (2004).

30. Kowalczyk, P. J. et al. Electronic size effects in three-dimensional nanostructures. Nano Lett. 13, 43 (2013).

31. Kokubo, I., Yoshiike, Y., Nakatsuji, K. \& Hirayama, H. Ultrathin Bi (110) films on Si (111) $\sqrt{3} \times \sqrt{3}-B$ substrates. Phys. Rev. B 91, 075429 (2015).

32. Lu, Y. H. et al. Topological properties determined by atomic buckling in self-assembled ultrathin $\mathrm{Bi}(110)$. Nano Lett. 15, 80 (2015).

33. Volovik, G. E.. The Universe in a Helium Droplet (Clarendon Press, 2003).

34. Schnyder, A. P., Ryu, S., Furusaki, A. \& Ludwig, A. W. W. Classification of topological insulators and superconductors in three spatial dimensions. Phys. Rev. B 78, 195125 (2008).

35. Yang, S. A., Pan, H. \& Zhang, F. Dirac and Weyl superconductors in three dimensions. Phys. Rev. Lett. 113, 046401 (2014).

36. Young, S. M. \& Kane, C. L. Dirac semimetals in two dimensions. Phys. Rev. Lett. 115, 126803 (2015).
37. Kane, C. L. \& Mele, E. J. Quantum spin hall effect in graphene. Phys. Rev. Lett. 95, 226801 (2005)

38. Fu, L. \& Kane, C. L. Topological insulators with inversion symmetry. Phys. Rev. B 76 045302 (2007).

39. Wei, Q. \& Peng, X. Superior mechanical flexibility of phosphorene and few-layer black phosphorus. Appl. Phys. Lett. 104, 251915 (2014).

40. Zhang, Y., Tan, Y.-W., Stormer, H. L. \& Kim, P. Experimental observation of the quantum Hall effect and Berry's phase in graphene. Nature 438, 201 (2005).

41. Nair, R. R. et al. Fine structure constant defines visual transparency of graphene. Science 320, 1308 (2008).

42. Kresse, G. \& Furthmuller, J. Efficient iterative schemes for $A b$ initio total-energy calculations using a plane-wave basis set. Phys. Rev. B 54, 11169 (1996).

43. Blochl, P. E. Projector augmented-wave method. Phys. Rev. B 50, 17953 (1994).

44. Solovyev, I. V., Dederichs, P. H. \& Anisimov, V. I. Corrected atomic limit in the local-density approximation and the electronic structure of $\mathrm{d}$ impurities in $\mathrm{Rb}$. Phys. Rev. B 50, 16861 (1994).

45. Grimme, S. Semiempirical GGA-type density functional constructed with a long-range dispersion correction. J. Comput. Chem. 27, 1787 (2006).

46. Heyd, J., Scuseria, G. E. \& Ernzerhof, M. Hybrid functionals based on a screened Coulomb potential. J. Chem. Phys. 118, 8207 (2003).

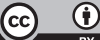

This work is licensed under a Creative Commons Attribution 4.0 International License. The images or other third party material in this article are included in the article's Creative Commons license, unless indicated otherwise in the credit line; if the material is not included under the Creative Commons license, users will need to obtain permission from the license holder to reproduce the material. To view a copy of this license, visit http://creativecommons.org/licenses/ by/4.0/

Supplementary Information accompanies the paper on the npj Computational Materials website (http://www.nature.com/npjcompumats) 